\title{
El nutricionista educador: Concepciones de estudiantes de la Licenciatura en Nutrición de la Universidad Nacional de Córdoba, Argentina
}

\author{
The nutritionist educator: conceptions of students from the Nutrition program of the National University of Córdoba, \\ Argentina
}

María Lis del Campo ${ }^{*}$ orcid.org/0000-0001-5669-5057

Erica María Martinich1 orcid.org/0000-0002-8015-8398

Alicia Navarro' ${ }^{\prime}$ orcid.org/0000-0003-3960-5456

Teresita Alzate ${ }^{2}$ orcid.org/0000-0002-3147-7990

1 Escuela de Nutrición, Facultad de Ciencias Médicas, Universidad Nacional de Córdoba. Córdoba, Argentina

2 Escuela de Nutrición y Dietética, Universidad de Antioquia. Medellín, Colombia

Del Campo ML, Martinich EM, Navarro A, Alzate T. El nutricionista educador: Concepciones de estudiantes de la Licenciatura en Nutrición de la Universidad Nacional de Córdoba, Argentina. Univ. Salud. 2017;19(2):215-225. DOI: http://dx.doi.org/10.22267/rus.171902.84

\section{Resumen}

Introducción: Los modos en que se han implementado las iniciativas educativas aparecen como zonas oscuras respecto de cómo contribuyen al mejoramiento de la situación alimentaria nutricional de la población argentina. Objetivo: analizar las concepciones sobre el nutricionista educador de estudiantes de Licenciatura en Nutrición. Materiales y métodos: Investigación exploratoria con preguntas cualitativas. Se realizaron 173 entrevistas semiestructuradas, analizando género, edad, año de cursado y los fundamentos del nutricionista como educador. Se utilizó muestreo intencional de máxima variación y análisis de contenido temático y de enunciación. Resultados: El 99\% de los estudiantes considera al nutricionista como educador. Se construyeron tres grandes categorías: la función educativa, según el plan de estudios; el rol de educador, en que la salud aparece como objeto del quehacer profesional mediado por la alimentación, o como referente socialmente legitimado para prescribir recomendaciones alimentarias; y las acciones educativas, mostrando diferencias y tensiones en las concepciones pedagógicas. Conclusiones: Los motivos por los que el nutricionista es un educador abarcan visiones trasmisionistas y algunas próximas a la educación problematizadora, fundamentadas desde la apropiación curricular y el quehacer profesional. Así, es posible visualizar ciertos rasgos que atraviesan la práctica de los egresados según las concepciones educativas que construyen desde que son estudiantes.

Palabras clave: Educación alimentaria y nutricional; educación superior; nutricionista. (Fuente: DeCS, Bireme).

\begin{abstract}
Introduction: The ways in which educational initiatives have been implemented appear as dark areas regarding how they contribute to the improvement of the nutritional situation of the Argentinean population. Objective: To analyze the conceptions about the nutritionist educator of students from the Nutrition program. Materials and methods: An exploratory research with qualitative questions was conducted. 173 semi-structured interviews were made where gender, age, year of study and the basics of the nutritionist as an educator were analyzed. Intentional sampling of maximum variation and analysis of thematic content and enunciation were used. Results: $99 \%$ of students consider the nutritionist as an educator. Three major categories were built: the educational function according to the curriculum; the role of educator, in which health appears as an object of professional activity mediated by food or as a socially legitimized reference to prescribe food recommendations; and educational actions,
\end{abstract}


showing differences and tensions in pedagogical conceptions. Conclusions: The reasons why the nutritionist is an educator include transmisionist visions and some others close to problematizing education based on the appropriation of curriculum and professional work. Thus, it is possible to visualize certain traits that go through the practice of graduates according to the educational concepts they build since they are students.

Keywords: Food and nutrition education; higher education; nutritionist. (Source: DeCS, Bireme).

\section{Introducción}

Argentina de manera similar a numerosos países de Latinoamérica, atraviesa un estadio avanzado de la transición demográfica, epidemiológica y nutricional(1-3), que ha implicado modificaciones en la jerarquización del grado de relevancia de las problemáticas alimentario-nutricionales y las enfermedades asociadas a ellas. Según la Encuesta Nacional de Nutrición y Salud (ENNyS) ${ }^{4)}$ se observa un aumento en las situaciones de sobrepeso y obesidad de las enfermedades crónicas no trasmisibles y la persistencia de baja talla y desnutrición oculta, con una disminución de las condiciones agudas de déficits.

A su vez, los planes y programas dependientes del Ministerio de Desarrollo Social y de Salud de la Nación han incorporado componentes de Educación Alimentaria-Nutricional (EAN), expresadas casi siempre en forma de conductas alimentarias de carácter individual para promover la salud y prevenir enfermedades: El Programa Educación Alimentaria del Plan Nacional de Seguridad Alimentaria (PNSA) en el marco de la Ley № 25724 en el año 2003(5) y también como componente de algunos programas del Ministerio de Salud de la Nación (Programa Nacional Municipios y Comunidades Saludables, Entornos Saludables, entre otros)(6); además de las numerosas campañas comunicacionales que se desarrollan, tendientes a la difusión de contenidos sobre la alimentación saludable cuyo eje principal está constituido por recomendaciones (mensajes) sobre esta temática. Sin embargo, los modos en que se han implementado las iniciativas educativas aparecen como zonas oscuras respecto de cómo contribuyen al mejoramiento de la situación alimentaria nutricional de la población.
En Argentina, el informe sobre Buenas Prácticas en Información, Comunicación y Educación en Alimentación y Nutrición (BP-ICEAN), realizado por la FAO, ha mostrado falencias $y$ contradicciones en la formación y prácticas educativas de los nutricionistas. El documento, entre los resultados principales, muestra que los objetivos y metodologías propuestas en los programas de ICEAN se basan en enfoques tradicionales que enfatizan la transmisión de información. En esos programas no se contemplan diagnósticos participativos, ni mecanismos claros y efectivos de evaluación. Además, quienes ejecutan las acciones en el terreno de la comunicación, consulta o consejería, no poseen la suficiente formación en el área(7).

Excepto el informe de FAO antes mencionado, es sumamente escaso lo que se conoce sobre la situación de la EAN en nuestro medio, dada la carencia de investigaciones sobre este tema. La Educación para la Salud, en que podría enmarcarse la EAN, si bien se considera un campo de conocimiento importante en las disciplinas del área de la salud humana, su desarrollo no ha sido coherente con esta premisa(8-10). Al investigar los referentes pedagógicos en intervenciones educativas en salud y nutrición en países de Iberoamérica se observan insuficiencias en los marcos teóricos que respaldan tales acciones, a la vez que un marcado énfasis en los contenidos enseñados y no en los procesos, o bien tensiones $\mathrm{y}$ contradicciones entre las concepciones y propuestas educativas analizadas(11). Resulta de vital importancia resaltar que tales hallazgos no pueden ser reducidos a datos estáticos 0 fragmentados, sino que deben problematizarse a la luz de las percepciones, experiencias, trayectorias institucionales y contextos de los actores sociales involucrados en los procesos en 
que se desarrollan las prácticas concernientes a la EAN.

Debido a la complejidad e implicaciones que implica toda acción educativa en alimentación y nutrición, se torna necesario comprender los posicionamientos, significados y prácticas de quienes se constituyen como educadores en este campo. Una instancia decisiva es la formación profesional, que incluye a las instituciones educativas formadoras, los docentes y los estudiantes, y los contextos en que estos se inscriben.

La formación profesional universitaria constituye el conjunto de conocimientos, habilidades y actitudes que los sujetos deben adquirir y desarrollar durante los estudios universitarios(11). En este proceso se ponen en relación elementos del campo de la ciencia, la tecnología y las humanidades sobre los que se estructuran las habilidades y actitudes específicas de una carrera profesional determinada, a la vez que cobra vigencia según las condiciones sociohistóricas en que se inscribe(12). Para ello, se establecen lineamientos teóricos y metodológicos que dan cuenta de una determinada concepción de la institución acerca de la educación y de los profesionales que se espera formar, que constituyen lo que se denomina currículo. Este elemento articula tanto la estructura de contenidos que se desarrollan a lo largo de la formación, como los principios ideológicos, políticos, psicopedagógicos y otros, en los que se fundamenta una institución educativa y normativiza las actividades a las que guía(13). Su diseño implica la toma de decisión y ordenamiento de los objetivos, propósitos, acciones y contenidos que orientarán las actividades de todos los actores sociales de una institución educativa. La síntesis instrumental de dicho ordenamiento corresponde a lo que se denomina Plan de Estudios y que, según Zabalza, constituye los "proyectos formativos que ofrecen las instituciones universitarias para la acreditación como profesionales de las diversas ramas del saber"(14).

En el caso particular de la Universidad Nacional de Córdoba, en el año 1985 se inició un período de reconocimiento y democratización de la carrera, gestada es sus inicios como Curso de Dietistas, que dio lugar a un proceso de revisión curricular cuyo producto fue el Plan de Estudios de la Licenciatura en Nutrición(15) vigente a la actualidad. Dicho plan incluye las siguientes áreas de formación multidisciplinaria: básica, socioeconómica, educación, salud pública, nutrición y alimentación. Desde su gestación se caracterizó por la impronta en la formación pedagógico didáctica de los profesionales, puesto que entre las funciones que componen el campo profesional se incluye la de educación y docencia que habilita el desempeño laboral en diferentes planos: la docencia en los diferentes niveles del sistema educativo formal -secundario, terciario y universitario de pre y posgrado-, la educación en nutrición y alimentación a nivel familiar y comunitario, dirección de cursos, carreras y escuelas con orientación en la temática, participación en la formulación de contenidos en nutrición y alimentación, capacitación de recursos humanos, dirección de unidades técnicas y de establecimientos de servicio alimentario(16). Por consiguiente, destina al área de formación en Educación un 5\%, 11\% y 13\% de la carga horaria correspondiente a $1^{\circ}$, $2^{\circ}$ y y $3^{\circ}$ año respectivamente, sin considerar los porcentajes destinados a otras áreas del currículum que la consideran como insumo para su desarrollo, ello marca una impronta en la formación pedagógico-didáctica y en la constitución de la identidad profesional de los futuros nutricionistas.

Según Almeida y Baldini Soares(17) el proceso educativo en salud, y por ende la EAN, en tanto fenómeno social, se construye a partir de la intencionalidad de los sujetos, los supuestos del educador, el posicionamiento de los estudiantes, las consecuencias en su formación y en la práctica profesional con la comunidad. Por esto, los marcos explicativos desde donde se entienda el quehacer educativo del nutricionista se desprenden en primera instancia, del entramado de significados y prácticas resultante de la interacción entre los estudiantes, los docentes y el plan de estudios, en un marco siempre espacial y temporalmente situado. 
Las concepciones de los estudiantes sobre el nutricionista como educador constituyen un inicio posible y también insoslayable, para una comprensión más acabada de la problemática expuesta en este apartado, y en consecuencia el objetivo de este trabajo. El mismo fue llevado adelante con estudiantes de la carrera de la Licenciatura en Nutrición de la Universidad Nacional de Córdoba (UNC) en el período lectivo correspondiente al año 2015.

\section{Materiales y métodos}

Esta investigación de tipo exploratorio o formativo(18), constituye la fase inicial de un estudio cualitativo profundizado y complementado en una etapa posterior.

La unidad de análisis fueron las concepciones de los estudiantes entendidas como esquemas de conocimiento, ideas, motivaciones y experiencias que aportan al proceso de representación del mundo y dan sentido a su realidad educativa(18), tomando como punto de partida sus perspectivas y ubicación como sujetos activos en el proceso educativo.

De los 1610 estudiantes inscritos en el año 2015 en la carrera de la Licenciatura en Nutrición de la Facultad de Ciencias Médicas de la UNC(19), participaron 173. La muestra se conformó a partir de la participación voluntaria. La delimitación muestral no fue numérica, sino que se buscó la abarcabilidad y diversidad(20), ya que la primera etapa fue de comprensión de las concepciones de los estudiantes, más que de la generalización de los resultados obtenidos. En esta dirección, se orientó la construcción de la muestra hacia la participación voluntaria de estudiantes con diferentes edades y grado de avance en la carrera.

Las respuestas se obtuvieron por medio de las aulas virtuales y sistema Guaraní de la UNC (sistema de gestión de alumnos que registra y administra todas las actividades académicas de la Universidad), por medio de mails en formato Google Drive, y de manera personal en formato papel. Fueron convocados en varias instancias, previo trabajo conjunto con el Área
Comunicación de la Escuela de Nutrición y con el Departamento de Informática de la Facultad de Ciencias Médicas de la UNC.

Como técnica de indagación se utilizó la entrevista semiestructurada, a fin de facilitar un primer abordaje de los supuestos de la investigación(21). Se indagaron los ejes centrales que orientaron la investigación: si considera que el Licenciado en Nutrición es un educador y los fundamentos de esta respuesta, expresados de manera abierta. Cabe señalar que el instrumento utilizado pertenece a un proyecto mayor, por lo que contiene un mayor número de ítems a indagar, de los que se tomaron para esta investigación la pregunta mencionada en el párrafo anterior y el porqué de la misma, ya fuera la respuesta negativa o afirmativa.

Para caracterizar el colectivo estudiantil se realizó el análisis de estadística descriptiva a través del programa Microsoft Excel 2013 considerando el elevado número de respuestas obtenidas. El análisis de contenido se llevó a cabo a través de análisis temático a las respuestas obtenidas en el cuestionario, lo cual consiste en hallar núcleos de sentido, cuya presencia o frecuencia denota estructuras de relevancia, valores de referencia y modelos de comportamiento subyacentes al discurso(21). Se efectuaron las siguientes etapas: lectura flotante del material; constitución del corpus y selección de las respuestas pertinentes. Se descartaron aquellas que por su contenido (tautologías, falta de pertinencia en relación al tema indagado) no posibilitaran dar respuesta a los objetivos de la investigación. También se determinaron las unidades de registro (palabras clave, frases y expresiones), la unidad de contexto, las formas de categorización y delimitación de los conceptos teóricos más generales que orientaron el análisis y construcción de categorías y subcategorías sistematizadas en la Tabla 1.

A fin de superar una mirada reduccionista del análisis, se utilizó análisis de la enunciación, entendiendo que las respuestas se enmarcan en un tipo particular de proceso comunicativo, en el cual no constituyen un producto acabado sino de creación de significados donde se encuentran 
contradicciones e incoherencias. Las entrevistas en tanto lugar en que se plasman los discursos, se reconocen también como una instancia al mismo tiempo espontánea y constreñida por la situación.

Tabla 1. Ejemplo resumido para el análisis de una de las categorías sobre porqué el nutricionista es un educador

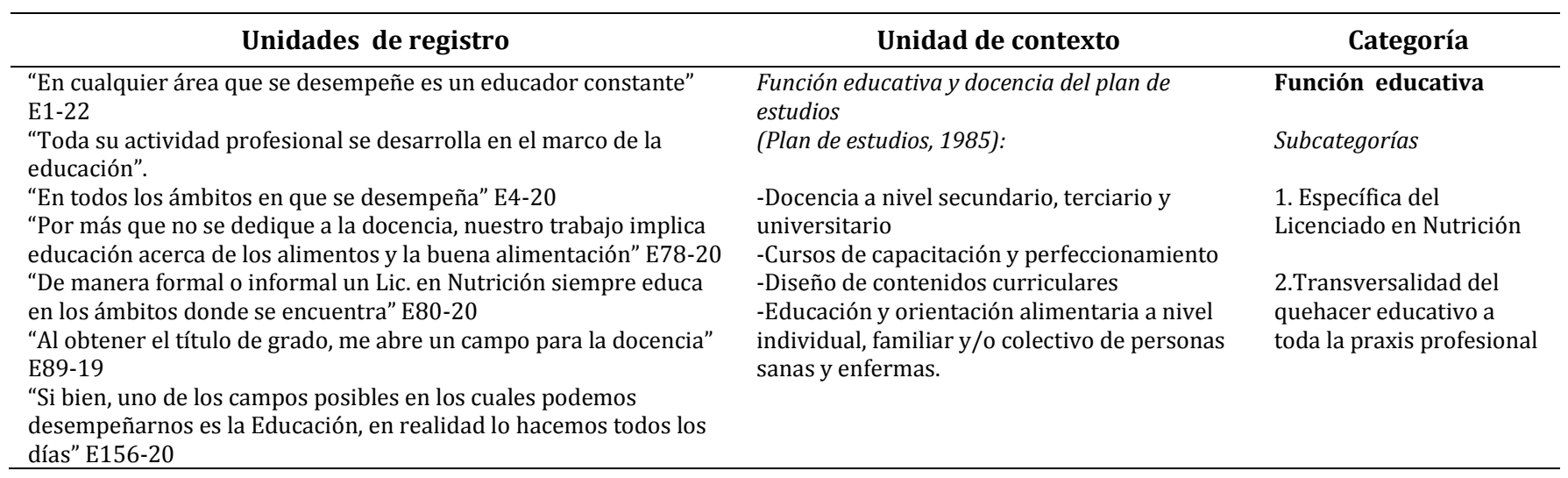

\section{Consideraciones éticas}

La presente investigación se enmarca dentro de los principios éticos básicos de la norma internacional de la Declaración de Helsinki y los criterios de conducción responsable de la investigación con seres humanos(22,23).

\section{Resultados}

Del total de estudiantes entrevistados la amplia mayoría pertenece al género femenino (91\%), en concordancia con el universo de estudiantes de la Escuela de Nutrición. La mayoría de los entrevistados $(58,4 \%)$ tienen entre 18 y 21 años (M0 = 19), y la edad de 20 años es el valor que se encuentra en el centro de la distribución $(\mathrm{Me}=$ 20). La muestra incluyó a estudiantes con distinto grado de avance en la carrera, con predominio de los matriculados en $2^{\mathrm{f}}$ año. La distribución se muestra en la Tabla 2.

Tabla 2. Distribución de estudiantes según año de cursado de la carrera

\begin{tabular}{lrr}
\hline \multicolumn{1}{c}{ Año de cursado } & n & \multicolumn{2}{c}{$\%$} \\
\hline 1er año & 20 & 11,56 \\
2do año & 85 & 49,13 \\
3er año & 36 & 20,81 \\
4to año & 20 & 11,56 \\
Prácticas profesionales & 7 & 4,05 \\
TIL & 5 & 2,89 \\
\hline *Tesis de Investigación para la Licenciatura & &
\end{tabular}

\section{El nutricionista como educador}

De manera general puede decirse que casi la totalidad de los estudiantes entrevistados (99\%) considera que el Licenciado en Nutrición es un educador, sin embargo, los argumentos que sostienen tal afirmación son diversos, heterogéneos y a veces imprecisos. El análisis de los fundamentos que argumentan por qué el nutricionista es un educador permitió la construcción de tres grandes categorías:

\section{La función educativa del nutricionista}

Para un número importante de estudiantes la docencia no es identificada como una función específica en el campo laboral tal como lo considera el plan de estudios de la carrera, sino como una dimensión de todo el quehacer profesional, que aparece transversalizando la práctica del nutricionista en un "área", "actividad", "ámbito" o "campo" ubicuo y permanente en el quehacer profesional, que puede ser ejercido de manera individual $o$ colectiva, ya sea en hospitales, comedores o escuelas.

Un número más reducido de estudiantes reconoce explícitamente la función educativa y docente, mencionándola según lo define y establece el plan de estudios. Esta función es concebida a nivel grupal en una comunidad/sociedad o a nivel individual. En este 
grupo predominan estudiantes en el primer año de la carrera, lo que posiblemente se relacione con la proximidad temporal al Ciclo de Iniciación (instancia evaluativa obligatoria para el ingreso a la Licenciatura) donde el análisis del Plan de Estudios es uno de los contenidos abordados.

\section{El rol de educador del nutricionista}

Aquí se materializa la percepción del quehacer profesional del nutricionista desde las miradas hacia el futuro, como aplicación de lo aprendido en la carrera. La dificultad al precisar campos o ámbitos de acción parece encontrar mayor especificidad cuando la función de educador es expresada como rol. Se entiende este término como una pauta habitual de comportamientos que se espera de un individuo que ocupa una posición en una estructura social(24). En consecuencia, las subcategorías derivadas no constituyen prácticas esperadas aisladas, sino que ponen de manifiesto dimensiones del contexto académico en particular, y sociocultural en general, que dan sentido a la conducta singular. En esta dirección, las tendencias que pueden observarse en las acciones educativas afloran asociadas a determinada finalidad $\mathrm{u}$ objeto. Así, las proyecciones de desempeño profesional emergen vinculadas a los conceptos de salud y enfermedad, lo cual no resulta sorprendente en vista de que desde hace décadas las prácticas de salud se vienen pensando en el marco de la Promoción de la Salud, en que la Educación para la Salud viene intentando distinguirse de las prácticas preventivistas tradicionales(25).

Considerando el contexto de formación de los estudiantes, es preciso reconocer que estos toman contacto con contenidos relacionados a este punto desde el primer año de la carrera. A su vez, la distinción entre la mirada preventivista, como acción anticipada a un daño o a evitar la aparición de enfermedades, y de la promoción de la salud enfocada en impulsar o fomentar medidas destinadas a aumentar la salud y el bienestar es un punto abordado, al menos desde el currículum, transversalmente en la carrera. Sin embargo, los estudiantes que mencionan este aspecto, asumen la intención de contribuir a la "prevención de enfermedades", "evitar problemas de salud" o a la disminución del "riesgo" de enfermar. Estas nociones parecen constituirse de manera un tanto difusa y como correlato de la reconocida definición de prevención en salud propuesta por Leavell \& Clarck en 1976(25), aludiendo a una acción anticipada que tenga como fin impedir la expansión posterior de la enfermedad. Otros estudiantes logran posicionarse desde la promoción de la salud, entendida como una estrategia que media la relación de las personas con el entorno y la elección personal con la responsabilidad social para crear ambientes saludables(26). De este modo, aparece como tarea inherente al nutricionista, el mejoramiento, cuidado o goce de la salud.

Otra modalidad en que los estudiantes significan el rol del nutricionista es como "referente social" en alimentación y nutrición; en este es posible observar dos tendencias: Una que alude al poder del conocimiento, en la que el nutricionista es concebido como quien "tiene en sus manos el conocimiento", o bien "educar a la sociedad" o la "responsabilidad" de que las personas adquieran conocimientos para mejorar su calidad de vida. Y la otra, que refiere al objeto del conocimiento en tanto el profesional es concebido como un "guía"; entendido desde una función prescriptiva acerca de la "manera correcta de alimentarse" y "ayudar a que comprendan la importancia de una correcta alimentación". Se explicita la idea de "decirles" a las personas qué comer, o de propiciar indicaciones para la adopción/incorporación de hábitos alimentarios saludables.

\section{El enfoque de las acciones del nutricionista}

Esta categoría se construyó considerando las conductas que los estudiantes mencionan para fundamentar por qué el nutricionista es considerado un educador. Se trata de comportamientos asociados al acto de educar. Se conformaron dos subcategorías de acuerdo a su proximidad con los enfoques pedagógicos que se trabajan y problematizan a lo largo de la carrera. Con base en los hallazgos, estas concepciones fueron: 
Trasmisionista; asociada a la idea de transferir conocimientos sobre alimentación para lograr cambios de conducta y mejorar el estilo de vida. Las expresiones utilizadas ("volcar", "trasmitir", "dar a conocer", "informar") y la finalidad que las orienta, permiten visualizar la proximidad con supuestos subyacentes sobre el enseñar, cercanos a lo que Paulo Freire(27) denominó teoría bancaria. Desde esta visión, el pedagogo brasileño cuestionó la educación tradicional, en tanto ésta reconoce al sujeto que aprende como un recipiente donde el docente deposita contenidos fragmentados y descontextualizados de la realidad. Desde otra concepción epistemológica, lo que Contento et al.,(28) denominaron Modelo de Educación en Nutrición de Conocimientos, Actitudes y Prácticas, en el que se asume que la exposición de las personas ante nueva información modificará sus actitudes $\mathrm{y}$ en consecuencia, sus comportamientos alimentarios, aludiendo la corrección y el reforzamiento de hábitos alimentarios, según estos sean saludables o no. Esta concepción se aproxima a la idea de enseñar como la práctica de incentivar la adhesión a una serie de recomendaciones alimentarias que se establecen de manera normativa (lo "saludable" o "correcto", un "buen plan alimentario") y son evaluadas y guiadas mediante reforzadores que implementa el profesional.

Crítica o reflexiva; relaciona la tarea educativa a la construcción de un vínculo entre el individuo/comunidad y el profesional, en el que se intercambian experiencias y saberes, y ambos se constituyen en sujetos activos de ese proceso. En asociación a esta concepción aparece como finalidad, ya no el cambio de comportamientos o la modificación de estilos de vida, sino la formación de "personas pensantes" con libertad de opinar, construir, proponer y crear. La reflexión y "acercar herramientas" son visualizadas como instancias de un "proceso dinámico" en el que los conocimientos, actitudes y prácticas pueden modificarse, pero también recuperarse, atendiendo a las pautas culturales, necesidades individuales y disponibilidad de recursos. Esta concepción suele ir acompañada de cuestionamientos a modos más tradicionales o impositivos del ejercicio de la tarea educativa, dejando entrever como foco de la EAN al sujeto y su realidad como soporte de la formación y fuente de conocimientos, propio de la educación problematizadora $(29,30)$.

\section{Discusión}

La finalidad de este trabajo fue caracterizar las concepciones del colectivo estudiantil sobre el nutricionista como educador. Por consiguiente, la estrategia metodológica utilizada en esta instancia constituye una aproximación exploratoria, que no permite dilucidar con profundidad el sentido de estas expresiones, pero sí abre un campo de interrogantes como guías orientadoras para el avance $\mathrm{y}$ profundización de la investigación sobre este tópico.

Los diferentes modos en los que es entendido el rol de educador y las concepciones pedagógicas que lo sustentan, muestran a lo largo de la carrera -y pese a ser un contenido abordado en las asignaturas pedagógicas $\mathrm{y}$ en otras relacionadas de manera más indirecta-, una fuerte presencia de elementos del modelo tradicional y conductista, frente a ciertas concepciones más críticas o problematizadoras. Esto da cuenta de que si bien en los programas de las asignaturas vinculadas a la EAN (Organización del Proceso Intelectual, Didáctica de la Nutrición y Educación Alimentaria y Nutricional)(31) las corrientes pedagógicas que se expresan de manera formal son las teorías del aprendizaje constructivistas y los modelos de intervención participativos, estos contenidos se traman con acciones que exceden la estructura planificada y constituyen significados y valores que se ponen en juego en la práctica de forma no intencionada. Todo esto da lugar a lo que se denomina currículum oculto, y abarca aquellos procesos de socialización que se desarrollan en la acción educativa ${ }^{(32)}$ y no están necesariamente especificados en el Plan de Estudios. En esta dirección, en una investigación previa con docentes de la Licenciatura en Nutrición de la UNC, emergió el valor otorgado a la tarea educativa, en que se manifestó discursivamente la adhesión al enfoque constructivista, o al menos participativo. No obstante, las 
contradicciones y tensiones en el sentido dado a aquellos términos revelaron la complejidad en su apropiación y dieron cuenta de una ruptura epistemológica que aún no se concreta con los enfoques tradicionales aún vigentes(33), como discursos y concepciones que se yuxtaponen y contradicen en las prácticas educativas que se construyen cotidianamente en las aulas.

Por otra parte, los hábitos alimentarios son percibidos como una vía decisiva para la prevención de enfermedades o la promoción de la salud. La asociación con las acciones o conductas con que se representa el acto de enseñar o educar, tal como se señaló en el ítem anterior, permiten visualizar que la difusión de información científica y las recomendaciones preventivas del cambio de hábitos constituyen visiones predominantes que hablan de los modos en que se articula el conocimiento científico y las prácticas en salud, en este caso por vía de la EAN. Pese a la escasez de investigaciones sobre el tema, estas debilidades y tensiones entre concepciones pedagógicas se ponen también de manifiesto en otros estudios sobre los referentes pedagógicos que subyacen a intervenciones educativas en salud y nutrición, así como en instituciones de nivel superior, incluyendo las carreras de Nutrición en Argentina(7-9).

Varios trabajos acuerdan que la Educación para la Salud es reconocida como un componente importante en la Salud Pública cuyo desarrollo no ha sido acorde a su relevancia( ${ }^{34,35)}$. En otras palabras: la función educativa o el rol de educador del nutricionista es reconocido de manera significativa, pero las motivaciones y concepciones pedagógicas subyacentes resultan diversas.

Carvalho(36) problematiza estas cuestiones examinando las potencialidades del modelo teórico de la Promoción de la Salud para el perfeccionamiento de las prácticas educativas en salud. Para ello, analiza documentos nodales en la formación profesional, como el Informe Lalonde(37), Alma Atta(38) y la Carta de Ottawa(39), los cuales plantean la necesidad de garantizar oportunidades y recursos que permitan alcanzar el máximo de salud, así como la noción de participación comunitaria y de una mayor actividad del público en las acciones de salud. Así, reconoce la necesidad de asumir la polisemia de tales términos y su análisis en el contexto en que son producidos como discurso, puesto que las consecuencias ético-políticas serán diferentes según la interpretación y los marcos de referencia que los oriente. En este sentido, las acciones vinculadas a la EAN en el imaginario estudiantil, privilegian recomendaciones individuales, priorizando la promoción de estilos de vida saludables. El énfasis discursivo en este punto hace suponer que se dejan de lado aspectos de carácter estructural como serían las condiciones o modos de vida, o bien una naturalización de ciertas desigualdades que atraviesan las condiciones de salud y nutrición.

Por su parte, Castiel(40) realiza una aguda crítica al modelo de la hiperprevención y advierte sobre el énfasis de la epidemiología en el control de comportamientos, entre los cuales se incluyen los hábitos alimentarios, para evitar riesgos en la salud. Esto es un sesgo de la Salud Pública que el autor reconoce como propio de las sociedades capitalistas e individualistas posmodernas. La visión de numerosos estudiantes acerca del nutricionista como guía o referente social al "enseñar" sobre los modos "correctos" de alimentarse para cuidar la salud, coincide con el modo paternalista y autoritario con que Castiel describe la visión prescriptiva de la prevención. Asimismo, Díaz et al.(9), enmarcan estas características en el modelo biomédico predominante y su acción normalizadora, intervencionista y medicalizante que atraviesan la concepción de salud y la configuración de un imaginario basado en el cambio de comportamientos y la adopción de hábitos considerados, por el profesional de la salud, como saludables.

Otro de los elementos condicionantes de la formación de educadores en salud es la interdisciplinariedad, y su importancia a la hora de desarrollar modelos formativos que sean concebidos por equipos en los cuales entren en relación diversas disciplinas(36). Esta falencia se advierte ya en la formación de grado, en el modo de organización de los espacios curriculares y en 
la interacción de profesionales formadores expertos en diversas áreas de la disciplina. En ese sentido, la carga horaria destinada a educación pierde peso si no puede ser pensada y enseñada en articulación con otras áreas/asignaturas, atendiendo a la complejidad de los objetos salud y nutrición, a la vez que en una profunda reflexión y esfuerzo sobre la articulación teoría, práctica profesional y compromiso social.

Los hallazgos de esta investigación reafirman la necesidad de ampliar y complejizar el abordaje en las categorías teóricas y metodologías a utilizar, puesto que es preciso explorar nuevas posibilidades en este campo que habiliten posicionamientos más críticos a la vez que aporten alternativas de transformación a la praxis pedagógica en el cotidiano académico.

\section{Conclusiones}

La presente investigación posibilitó una primera aproximación a las concepciones sobre el nutricionista como educador de estudiantes de la Licenciatura en Nutrición, entendiendo a este grupo como uno de los actores involucrados en dicho escenario. El nutricionista es reconocido como educador por los estudiantes de la carrera, sin embargo, las concepciones que sustentan tal afirmación muestran diferentes modos de entenderla, que se vinculan con las posiciones, expectativas $\mathrm{y}$ trayectorias personales $\mathrm{y}$ académicas desde las cuales se piensan como futuros profesionales.

El curriculum universitario y los itinerarios formativos que se trazan guardan relación con el proceso de legitimación para el ejercicio profesional. En este sentido, la concepción del rol del nutricionista como educador posibilita pensar la articulación entre las expectativas de desempeño profesional y el contexto en el que se inscriben. La revisión de este rol a la luz de los hallazgos amerita repensar las concepciones de salud, nutrición y educación abordadas en el plan de estudios, así como sus relaciones, para comprender cómo los estudiantes son producidos como sujetos educadores en alimentación y nutrición.
A cada acción educativa en alimentación y nutrición subyace una determinada concepción de la salud, la educación, el sujeto y la sociedad, por lo que se torna necesario redimensionar la tarea educativa en el marco de la complejidad de los contextos actuales desde una mirada que abarque sus múltiples aristas, y posibilite el abordaje de problemáticas reales.

Así como para mejorar la situación alimentaria nutricional es necesario primero identificar aquellas problemáticas que la atraviesan, para efectuar recomendaciones acerca de las necesidades de la EAN es necesario comprender su realidad y su contexto.

Las contradicciones y tensiones en el sentido dado al rol del nutricionista como educador revelan la complejidad -a veces precaria- en su apropiación, y la necesidad de un análisis más profundo de las prácticas educativas que se desarrollan en el ámbito universitario, todo esto con el fin de aportar a la construcción de iniciativas que posibiliten la ruptura epistemológica con los enfoques tradicionales aún vigentes.

Finalmente, los hallazgos de esta investigación reafirman la necesidad de ampliar y complejizar el abordaje en las categorías teóricas y metodologías a utilizar, puesto que es preciso explorar nuevas posibilidades en este campo que habiliten posicionamientos más críticos a la vez que aporten alternativas de transformación a la praxis pedagógica en el cotidiano académico.

Conflicto de intereses: ninguno declarado.

\section{Referencias}

1. Duran P. Transición epidemiológica nutricional o "el efecto mariposa". Arch Argenitno Pediatría. 2005;103:195-7.

2. Orden A, Torres M F, Luis M A, Cesani M F QFA y OE. Evaluación del estado nutricional en escolares de bajos recursos socioeconómicos en el contexto de la transición nutricional. Arch Argenitno Pediatría. 2005;103:205-11.

3. Barría, P y Amico H. Transición Nutricional: una revisión del perfil latinoamericano. Arch Latinoam Nutr. 2006;56(1):3-11.

4. Ministerio de Salud de la Nación. Encuesta Nacional de 
Nutrición y Salud. Argentina: Ministerio de Salud de la Nacion; 2007.

5. Ministerio de Desarrollo Social. Políticas Sociales del Bicentenario. Un Modelo Nacional y Popular [Internet]. Argentina: Ministerio de Desarrollo Social; 2010. Disponible en: http://www.desarrollosocial.gob.ar/pnsa

6. Ministerio de Salud de la Nación. Programas y planes [Internet]. 2016 [citado 30 de mayo de 2017]. Disponible en: http://msal.gob.ar/

7. Organización de las Naciones Unidas para la Alimentación y la Agricultura. Buenas Prácticas en Información, Comunicación y Educación Alimentario Nutricional en la República Argentina [Internet]. Argentina: FAO; 2011. Disponible en: http://fao.org/docrep/019/as486s/as486s.pdf

8. Álzate Yepes T. Desde la educación para la salud hacia la pedagogía de la educación alimentaria y nutricional. Perspect en Nutr Humana. 2006;16:21-40.

9. Díaz P, Peñaranda F, Cristancho S, Caicedo N, Garcés M, Alzate $\mathrm{M}$, et al. Educación para la salud: perspectivas y experiencias de educación superior en ciencias de la salud, Medellín, Colombia. Rev Fac Nac Salud Pública. 2010;28(3):221-30.

10. del Campo, ML; Messler, M \& Navarro A. Educación alimentaria-nutricional (EAN) en la enseñanza primaria municipal de Córdoba: una experiencia en investigación-acción participativa (IAP). Diaeta. 2010;28:15-22.

11. Bautista J de L. Ciencia y Humanismo en la Formación Profesional Universitaria. En: I Congreso Iberoamericano de Ciencia, Tecnología Sociedad e Innovación "Ciencia, Tecnología e Innovación para el desarrollo en Iberoamérica [Internet]. Mexico DF.; $2006 . \quad$ Disponible en: www.oei.es/historico/memoriasctsi/mesa4/m04p21.p df

12. Pansza GM. "Elaboración de programas", en Operatividad de la didáctica. Vol. 1. Gernika, editor. México; 2005. 1-19 p.

13. Coll C. Los fundamentos del currículum. En: Mexicana P, editor. Psicología y currículum. Mexico; 1992.

14. Zabalza M. Curriculum universitario innovador.inuevos planes de estudio en moldes y costumbres viejas? En: De EP de EUPV, editor. Jornada de Formación de Coordinadores P-E [Internet]. Valencia; 2003. p. 2. Disponible en: http://www.upv.es/europa/doc/Articulo Zabalza.pdf

15. Navarro A. Un nuevo perfil profesional. Hoy la Universidad, Septiembre de 1987,Universidad Nacional de Córdoba.

16. Médicas F de C. Plan de Estudios de la Liencenciatura en Nutrición. Córdoba, Argentina; 1986.

17. Almeida A, Soares C. Health education: analysis of its teaching in undergraduate nursing courses. Rev Lat Am Enfermagem [Internet]. 2011;19(3):614-21. Disponible en: http://www.scielo.br/scielo.php?pid=S010411692011000300022\&script=sci_arttext

18. Ulin, PR; Robinson, ET y Tolley E. Investigación aplicada en salud pública. Métodos cualitativos.
Whashington, D.C.; 2006.

19. Universitarias. $\mathrm{P}$ de estadística. Anuario Estadístico 2015. Córdoba, Argentina; 2015.

20. Miralles Martínez, P. y Alfageme González M. Análisis de las concepciones del alumnado de Educación Infantil sobre la familia. Rev Didáctica las Ciencias Exp y Soc. 2010;24:45-61.

21. De Sousa Minayo M. La artesanía de la investigación cualitativa. 1er ed. Editorial L, editor. Buenos Aires; 2009.

22. Asociación Médica Mundial. Declaración de Helsinki. 2008.

23. Emanuel EJ. What Makes Clinical Research Ethical? Jama. 2000;283(20):2701.

24. Berger, P y Luckmann T. La construcción social de la realidad. 18va reimp. Amorrortu, editor. Argentina; 2003.

25. Czeresnia D. El concepto de salud y la diferencia entre prevención y promoción. En: Editorial L, editor. Promoción de la salud Conceptos, reflexiones y tendencias. 1er ed. Argentina; 2006. p. 47-63.

26. Restrepo S. La promoción de la salud y sus aportes a la educación en alimentación y nutrición. Univ Antioquia. 2005;23(1):110-7.

27. Freire P. Pedagogía del oprimido. 3er ed. Editores SX, editor. Buenos Aires; 2008. 29 p.

28. Contento IR, Balch GI. The effectiveness of nutrition education and implications for nutrition education policy. J Nutr Educ. 1995;27:277-422.

29. Davini M. Métodos de enseñanza: Didáctica general para maestros y profesores. 1er ed. Santillana, editor. Buenos Aires; 2008.

30. Navarro, A y del Campo M. Hacia una didáctica de la nutrición. Herramientas pedagógicas para la Educación Alimentaria Nutricional. Brujas, editor. Córdoba, Argetnina; 2015.

31. Escuela de Nutrición. Asignaturas [Internet]. 2017 [citado 19 de junio de 2017]. Disponible en: http://www.nutricion.fcm.unc.edu.ar/index.php/asign aturas

32. Diaz Barriga Á. La educación en valores: Avatares del currículum formal, oculto y los temas transversales. Rev Electrónica Investig Educ [Internet]. 2006;8(1):1$15 . \quad$ Disponible en: http://www.scielo.org.mx/scielo.php?script=sci_arttex t\&pid=S1607-40412006000100001\&lng=es\&nrm=iso

33. Navarro, A; del Campo M. El nutricionista educador: visión de docentes que lo forman. Arch Latinoam Nutr. 2015;65(1):103-4.

34. Gazzinelli, MF; Gazzinelli, A; Reis D dos; PC. Educação em saúde: conhecimentos, representaçõessociais e experiências da doença. Cad Saude Publica. 2005;21(1):200-6.

35. Peñaranda, F; Giraldo, L; Barrera, LH; Castro E. Significados de la educación para la salud en la Facultad Nacional de Salud Pública de la Universidad de Antioquia (2011-2012). Rev Fac Nac Salud Pública. 2014;32(3):364-72.

36. Carvalho S. Promoción de la Salud, "empowerment" y educación: una reflexión crítica como contribución a la reforma sanitaria. Salud Colect. 2008;4(3):355-347. 
37. Lalonde M. A new perspective on the Health of Canadians: a working document. Ottawa, Canada; 1974.

38. Organitation PAH. Declaration of Alma-Ata. En AlmaAta, URSS; 1978. Disponible en: http://www.paho.org/hq/index.php?option=com_doc man\&task=doc_view\&gid $=19004 \&$ Itemid $=270$

39. Organization WH. Ottawa Charter for Health Promotion. Canada; 1986.

40. Castiel L. La Salud Persecutoria. Rev Saude Publica. 2007;41(3):461-6. 